\title{
The First Meeting of Hand Hygiene Campaigning Nations: WHO World Alliance for Patient Safety, August $2007^{1}$
}

\section{Executive summary}

The WHO Guidelines on Hand Hygiene in Health Care (Advanced Draft) recommend that national governments:

- Make improved hand hygiene adherence a national priority and consider provision of a funded, coordinated and implemented programme of improvement.

- Support strengthening of infection control capacities within health-care settings.

- Promote hand hygiene at the community level to strengthen both self-protection and the protection of others.

Since the inception of the First Global Patient Safety Challenge of the WHO World Alliance for Patient Safety, twenty-two national or sub-national campaigns to address low compliance with hand hygiene by health-care workers have been identified.

In August 2007, twenty-one representatives from sixteen of these countries attended the first global gathering of campaigning nations in Geneva.

One of the key objectives of the meeting was to explore the opportunities for strengthening the global response to health care associated infections through the solidarity of a formal partnership of nations, each with a common aim - to address HAI through a focus (albeit not exclusive) on better hand hygiene compliance by health care workers.

The meeting facilitated in-depth sharing of the history and progress of national/sub-national campaigns. What emerged was a range of campaigns having several common features and differing in their extent and stage of maturity. The challenge posed by all countries concerned sustainability, with a consensus that this will only be achieved if hand hygiene improvement is integrated with existing infection control and patient safety activity. Hand hygiene and patient safety were described as forming an axis of health policy and clinical practice.

The meeting resulted in general support for strengthening the partnership of nations, with the WHO World Alliance for Patient Safety playing a central, supportive and facilitative role. Greater partnership between nations was seen as important in sustaining improvement and potentially assisting with spread-

\footnotetext{
${ }^{1}$ Available at: http://www.who.int/patientsafety/events/07/ps_meeting_report_geneva_29august.pdf.
} 
ing the aspirations and objectives of the First Global Patient Safety Challenge beyond its current reach, particularly to developing countries, moving the world towards cleaner, safer health care.

One of the chief outcomes of the meeting was the agreement to establish a small working group of representatives to further scope the way forward for national campaigners. The working group will develop a proposal for consideration by the World Alliance for Patient Safety.

What is clear is that these nations constitute the beginnings of a global partnership or movement. As a result of national campaigns, many thousands of hospitals are taking action to change systems which have historically not supported optimal hand hygiene compliance. This poses an opportunity to disseminate knowledge and experiences, learn more about the critical success factors behind promotion strategies and at the same time harmonize these efforts to improve the safety of care. This as yet untapped global partnership further opens the door to future large scale implementation of effective infection control interventions per se. As one of the attendees concluded, strengthening the collaboration between country campaigning nations could become a key step on the road to a global patient safety family, where collective wisdom results in action which benefits all.

\section{Participants}

The target audience of the meeting included representatives from countries which have already initiated or are in the preparation phase of national or sub-national hand hygiene improvement campaigns. In addition, the Core Technical Group of the First Global Patient Safety Challenge were in attendance (see Annex 1).

Additional attendees were comprised of members of the secretariat of the WHO World Alliance for Patient Safety and representatives from pilot and complementary test sites.

\section{Background briefing paper}

All attendees were provided with a briefing paper, ahead of the meeting, outlining the background to the World Alliance for Patient Safety (WAPS), the First Global Patient Safety Challenge (GPSC1) and the WHO Guidelines on Hand Hygiene in Health Care (Advanced Draft) (referred to as The Guidelines).

\section{Goals of the meeting}

The goals of the meeting as listed in Section 1 of the Briefing Paper:

(i) to establish the different approaches and extent of current campaigns;

(ii) to determine best measurement parameters;

(iii) to determine how evaluation and other outcome-related data might be used;

(iv) to agree the future relationship between WHO/University Hospitals Geneva and national campaign countries;

(v) to determine the feasibility and interest in establishing a formal "Club" of hand hygiene campaign countries;

(vi) to determine the potential role of campaigning countries in scale-up, spread and sustainability at a global level;

(vii) to determine how best to share knowledge and improve safety of patients through national campaigning. 